\title{
ENCLAVES DE LA TRANSICIÓN Y DEMOCRACIA CHILENA*
} Enclaves of the Transition and Chilean Democracy

\author{
PETER M. SIAVELIS
}

Wake Forest University

\begin{abstract}
RESUMEN
Algunas de las dificultades del gobierno de Michelle Bachelet y la crisis más generalizada del gobierno de la Concertación han estado cada vez más vinculadas a variables de liderazgo, al agotamiento de la coalición con respecto a sus ideales básicos, o a la necesidad de diversos tipos de reformas institucionales. Este trabajo contempla la posibilidad de que el modelo mismo de transición, que ha sido considerado como tan exitoso, podría ser el origen de las dificultades que hoy invaden tanto la Concertación como la democracia chilena en general. Además, argumenta que la interacción del sistema electoral y la estructura de la competencia postautoritaria han obligado a una política elitista que ayuda a explicar las dificultades actuales que sufre la Concertación, y ha forzado una crisis generalizada de representación. Para elaborar este argumento el autor se basa en el trabajo de Manuel Antonio Garretón, sobre los enclaves autoritarios, señalando que hay ciertos enclaves de la transición que dificultan el desarrollo de una democracia representativa de calidad. Estos enclaves de transición incluyen: el cuoteo, control de la élite en la selección de candidatos y la política electoral, dominación de los partidos en la política, formulación de políticas elitista y extrainstitucional, y la intocabilidad del modelo económico heredado del gobierno de Pinochet.
\end{abstract}

Palabras clave: Chile, Michelle Bachelet, enclaves de transición, Concertación, enclaves autoritarios.

\begin{abstract}
Some of the difficulties of Michelle Bachelet Government and the more generalized crisis of Chile's governing Concertación coalition have increasingly been tied to leadership variables, the exhaustion of the coalition with respect to its core ideals, or the need for various types of institutional reform. This paper considers the possibility that the very model of transition which has been lauded as so successful might actually be at the root of the difficulties that today plague both the Concertacion and Chilean democracy more generally. This paper argues that the interaction of the electoral system and the structure of post-authoritarian competition have forced an elitist form of politics that helps to explain the current difficulties plaguing the Concertacion coalition and fueled a generalized crisis of representation. To make this argument it builds on the work of Manuel Antonio Garretón concerning authoritarian enclaves, arguing that there are certain enclaves of the transition that get in the way of the development of a high quality representative democracy. These transitional enclaves include: el cuoteo, elite control of candidate selection and electoral politics, party dominated politics, elitist and extra-institutional policymaking, and the untouchability of the economic model inherited from the Pinochet government.
\end{abstract}

Key words: Chile, Michelle Bachelet, transitional enclaves, Concertación, authoritarian enclaves.

* $\quad$ El autor agradece a David Altman, Rosana Castiglioni, María de los Ángeles Fernández, Bonnie Field, Robert Funk, Fernando Meza, Kirsten Sehnbruch y dos referís anónimos por sus útiles comentarios sobre este artículo. Traducido por Pilar Gianinni. 


\section{ENCLAVES DE LA TRANSICIÓN Y LA DEMOCRACIA CHILENA}

A lo largo de la década de 1990 Chile ha sido sistemáticamente reconocido como un país que experimentó una transición democrática modelo y como una de las democracias más exitosas en América Latina. Con el paso de los años, algo del brillo se ha apagado en relación al modelo chileno. La mala gestión del gobierno, la percepción de un aumento de la corrupción y la escalada de protestas durante la administración socialista de la Presidenta Michelle Bachelet han dado lugar a un aumento de críticas hacia la Concertación, coalición que ha gobernado Chile desde el retorno a la democracia en 1990. Algunos han culpado el estilo de liderazgo de Bachelet, mientras que otros han vinculado los problemas de su gobierno más claramente con la propia Concertación. Habitualmente, académicos y los medios de comunicación han relacionado los problemas de la coalición a una "ideología de gobiernismo", aludiendo a que la Concertación se encuentra "agotada" o sugiriendo que el gobierno sufre de una "fatiga de material", "desgaste", todo lo cual puede ser señal del "fin de ciclo" de los gobiernos de la Concertación. ${ }^{1}$ Estas críticas se centran principalmente en la noción de que la Concertación se ha quedado sin ideas porque ha permanecido demasiado tiempo en el poder. Otros han discutido los elementos institucionales que se han interpuesto en el camino de la consolidación de una democracia representativa de alta calidad en el país. Altman (2006), por ejemplo, encuentra debilidades en las áreas de participación ciudadana, la rendición de cuentas y mecanismos de control, y propone reformas al sistema institucional chileno con el fin de remediar algunas de estas debilidades del sistema político. Junto con estas dificultades, la opinión pública y las explicaciones académicas indican una crisis generalizada de representación y una disminución de la identificación de la ciudadanía con los partidos políticos, que han sido tradicionalmente los principales interlocutores de la política chilena (véase Luna, 2008 y Siavelis, 2008).

Pocos han considerado la posibilidad de que el modelo mismo de transición, que ha sido considerado como tan acertado, en realidad podría ser el origen de las dificultades que afectan tanto a la Concertación como a la democracia chilena actual. Para elaborar este argumento, el trabajo desarrolla la idea de enclaves de la transición. En base al trabajo de Manuel Antonio Garretón (2003), quien sostuvo que ciertos enclaves autoritarios, heredados del régimen autoritario, interfirieron con el funcionamiento óptimo del sistema democrático; esta investigación argumenta que la interacción del sistema electoral y la estructura de competencia postautoritaria han creado un conjunto similar de enclaves de transición. En particular, el trabajo se centra en cinco enclaves de transición: el cuoteo, el control de la élite en selección de candidatos y la política electoral, dominación de los partidos en la política, formulación de políticas elitista y extrainstitucional, y la intocabilidad del modelo económico heredado del gobierno de Pinochet. Además, el trabajo sostiene que, debido a que este conjunto de instituciones, comportamientos, valores y creencias se basan en el sistema electoral legislativo y el contexto profundamente enraizado de la política partidista postransición, será muy difícil, si no imposible, eliminar sin una reforma electoral o un choque político exógeno sustancial la dinámica existente de la política partidaria.

1 Para un detalle de este argumento véase María de los Ángeles Fernández, “Concertación: artistas de un debate obligado", manuscrito no publicado, p. 2. 
Este trabajo procede de la siguiente forma. La primera sección analiza el argumento original de Garretón sobre enclaves autoritarios y discute los paralelos entre enclaves autoritarios y de transición, enfatizando en este último, a fin de seguir refinando el concepto de enclave político. La segunda sección explora los cinco enclaves de transición, detallando por qué cada uno de ellos es un enclave de acuerdo a la definición de los parámetros establecidos en este artículo, y además explora por qué cada uno de ellos es difícil de eliminar. La conclusión analiza los desafíos que se enfrentan en la elaboración de una nueva forma de la política posterior a la transición.

\section{ENCLAVES AUTORITARIOS Y ENCLAVES DE TRANSICIÓN}

La idea de los enclaves de transición se basa en el trabajo pionero de Manuel Antonio Garretón. Cuando se discutía de los desafíos planteados en la transición democrática en Chile, el autor definió enclaves autoritarios como ciertos elementos "del régimen anterior" que persisten "en el régimen democrático" (2003: 47-8). Subrayó que estos eran institucionales (leyes y la Constitución), socioculturales (valores autoritarios, etc.), basados en los actores (las Fuerzas Armadas o actores de veto), o ético-simbólicos (problemas de derechos humanos no resueltos). Estos enclaves, para Garretón, interfieren en la consolidación de la democracia. Este trabajo sostiene que un conjunto similar de enclaves dejados durante la transición han obstaculizado la plena consolidación de una democracia de calidad en Chile y explican, en parte, las dificultades que sufre la Concertación (Garretón, 2003).

Esta discusión genera interrogantes en torno a la definición, algunas relacionadas con la conceptualización original de Garretón de los enclaves autoritarios y en relación con los enclaves de transición en sí. Un esfuerzo para definir cuidadosamente los enclaves de transición y algunas advertencias de definición podrían contribuir a resolver ciertos cuestionamientos relacionados con el concepto y contribuir a una conceptualización más eficaz de los enclaves políticos de forma global.

En primer lugar, para que un enclave sea denominado como tal debe haber nacido de la dinámica política de un modelo político previo. El abrupto final del régimen militar chileno, por medio de plebiscito de 1988, proporcionaba un punto final concreto para el régimen militar chileno y una delimitación clara de un periodo político del cual las élites podrían heredar un legado. Garretón se refiere a los artefactos políticos heredados de un régimen militar, claramente delimitado, en términos temporales. Sin embargo, para los enclaves de transición, la periodicidad de un cambio de modelo político es menos clara y debe ser definido. Este trabajo considera, entonces, que la transición chilena comienza con el plebiscito de 1988 y termina en el 2005 con las reformas a la Constitución de Pinochet que eliminan la mayor parte de los elementos no democráticos de la Constitución. Después de estas reformas, Chile finalmente encontró las normas básicas de la democracia establecidas por Linz y Stepan, dado que "los poderes Ejecutivo, Legislativo y Judicial generados por la nueva democracia" ya no tienen que "compartir el poder con otros órganos de jure" (1996:3). Por lo tanto, los enclaves de transición han nacido a partir de modelos políticos y las interacciones consolidadas durante este periodo, es decir, entre 1988 y 2005. 
En segundo lugar, la conceptualización original de Garretón sobre los enclaves funciona bastante bien cuando se trata de enclaves constitucionales o institucionales de los regímenes militares. Sin embargo, ampliando el concepto para incluir también la conducta o los enclaves simbólicos, el término de enclave se convirtió en algo estirado y menos útil porque Garretón no diferenció los enclaves de otras posibles formas de actividad política informal. Por ejemplo, se podría argumentar que algunos de estos enclaves son simplemente las instituciones informales del tipo identificado por Helmke y Levitsky (2004). ¿Cuál es entonces la diferencia entre una institución informal y un enclave? La respuesta más simple es que un enclave puede, de hecho, ser una institución informal, no existe necesariamente una contradicción entre un concepto y otro. No obstante, no todas las instituciones informales son enclaves. Para diferenciar entre los tipos de instituciones informales (es decir, aquellos que pueden ser considerados enclaves y las que no), es útil volver a la etimología de la palabra enclave. Sus orígenes se remontan al latín, inclavare o encerrado [en+CLAVIS (llave)]. Mientras que las instituciones informales abundan en muchos sistemas políticos, un enclave es un tipo formal o informal para el cual hay fuertes obstáculos prácticos o políticos para su transformación. Para el caso de los enclaves de transición, en el presente trabajo dos variables primarias son la causa de la perpetuación de cada uno: el sistema binominal y el modelo firmemente enraizado de competencia política postautoritaria. De hecho, la propia interacción de estas dos variables hace que sea, prácticamente, imposible erradicar los enclaves excepto por un tsunami político generalizado o una reforma del sistema electoral.

Este debate plantea un tercer punto de definición. Los enclaves preservan eficazmente las prerrogativas de los actores políticos del período anterior y éstos tienen un incentivo para mantenerlos (ya se trate de un interés propio o un incentivo para lograr metas más elevadas). Los enclaves identificados por Garretón preservan el poder y las prerrogativas de las fuerzas aliadas con el régimen militar y la derecha. Sin embargo, los enclaves de la transición (intencionales o no) diferencian el poder y la influencia de las elites democráticas enraizadas versus aquellos aliados con el régimen autoritario. Por lo tanto, si bien algunos de los enclaves de transición discutidos aquí, y en particular la dinámica de la selección de candidatos, podrían bien ser considerados como un enclave autoritario, ya que emergió del sistema electoral autoritario, pero hay una diferencia importante que distingue a los enclaves autoritarios de aquellos de transición. La dinámica del proceso de selección de candidatos es un enclave que protege el poder y la influencia de las elites de transición. En este sentido, aunque el sistema binominal es un enclave autoritario, todo el proceso y la dinámica de la selección de candidatos que emergió como consecuencia puede considerarse mejor como un enclave de transición.

En resumen, y siguiendo a Goertz (2005), este trabajo utiliza una conceptualización de enclaves sobre la base de una lógica, necesaria y suficiente. ${ }^{2}$ Con el fin de ser considerados enclaves, instituciones formales e informales y los patrones de comportamiento político deberán tener los siguientes elementos:

2 Esto es distinto de la lógica de " family resemblance", utilizada por Goertz en el sentido de que todas estas condiciones deben estar presentes para que un enclave exista. Mirar el Capítulo 2 en Goertz. 
1) Tener un origen en la dinámica de un modelo político previo,

2) Ser difícil de desplazar por motivos prácticos o institucionales, y

3) Proteger o preservar los intereses políticos de los principales actores que tienen un interés en mantenerlos.

Hay, por supuesto, una práctica más importante y, ciertamente normativa, que es significativo establecer aquí. Los enclaves de la transición, en la mayoría de los casos, no fueron impuestos por el régimen militar. En este sentido, gozan de una mayor legitimidad democrática y son cualitativamente diferentes de enclaves autoritarios. Sin embargo, existen ciertas similitudes entre los dos tipos de enclaves. Los enclaves de transición, al igual que los autoritarios, son multidimensionales (ellos constan de instituciones, formas de pensar y maneras de actuar, etc.). Además, ambos tipos no son totalmente negativos y, en cierto modo, hacen una contribución positiva a la consolidación de la democracia. Por ejemplo, se podría argumentar, en algunos sentidos, que la presencia y la influencia de los militares durante la transición en realidad contribuyeron a la democratización garantizando los intereses fundamentales de los posibles actores de veto. Del mismo modo, es importante reconocer que muchos de los enclaves de transición jugaron un papel muy positivo en el éxito de los compromisos de transición democrática.

Con estas definiciones y advertencias conceptuales establecidas, el análisis se centra ahora en aquellos enclaves de transición. A continuación se presenta una breve discusión de por qué cada uno puede ser considerado un enclave y por qué es tan difícil su erradicación.

\section{El cuoteo:}

La Concertación, coalición que ha gobernado Chile desde el retorno de la democracia, se basa en una elaborada forma de distribución del poder que se conoce como "cuoteo". Los detalles de esta negociación incluyen una cuidadosa división de carteras ministeriales entre sus partidos constituyentes. Los subsecretarios han sido, en general, de un partido diferente (y usualmente de un sector ideológico distinto) que el del ministro. Si bien no existe un acuerdo formal de tal arreglo, la institución informal de entrada de los partidos, extendida en la toma de decisiones ministerial, ha proporcionado un incentivo para el mantenimiento de la coalición. Lo que es más, a lo largo de los ministerios, y en particular en los ministerios "políticos", cada administración postautoritaria procuró proporcionar una representación completa de los partidos políticos que integran la Concertación en personales de nivel superior. Estos acuerdos caracterizan la mayor parte de la administración pública e incluso se extienden a la distribución de los escaños de los candidatos legislativos. Las diferentes facciones partidistas, dentro de la coalición, también apelan al presidente para situar a una serie de funcionarios de cada una de las facciones en posiciones de poder en toda la rama ejecutiva y en otras áreas en las que el presidente realiza nominaciones. ${ }^{3}$

3 “La 'dedocracia' de Lagos", 12 marzo 2000, El Mercurio, edición on line (www.emol.cl) y "Presiones por los cupos de subsecretarios", 1 febrero 2000, La Tercera, edición on line (www.latercera.cl). 
El cuoteo es una de las claves del éxito de la transición democrática. Esta forma de distribución de ministerios y el trabajo compartido refuerza la confianza al asegurar la entrada de los partidos en la adopción de decisiones gubernamentales. La dispersión de gabinete ha prevenido la verticalización de ministerios particulares en instituciones de distribución de patronazgo para un partido. El patronazgo y la influencia han sido dispersados en toda la coalición, extendiendo así las ganancias del éxito electoral, y transformando lo que podría ser un juego de suma cero de competencia partidaria en un juego de suma positivo. (Rehren, 1992). El cuoteo fue diseñado como una institución destinada a garantizar la amplia y completa representación de todos los partidos de la coalición. Sin esta representación y voz, los partidos habrían tenido pocos incentivos para permanecer leales a la coalición y probablemente se habría derrumbado, y Chile no sería el ejemplo de éxito de la transición democrática que es hoy.

Sin embargo, a pesar de la posición central que tiene el cuoteo en el éxito de la transición democrática, este arreglo ha sobrevivido su utilidad. El cuoteo es a menudo visto burlescamente por la ciudadanía y existe la impresión de que no se conceden posiciones ministeriales basadas en los talentos o en la experiencia necesaria para ser ministro, sino que en las exigencias políticas que establecen los partidos. Los chilenos cada vez más ven el cuoteo como una forma de politiquería y como un modo de asegurar posiciones políticas para los políticos, algunos de los cuales no han tenido éxito en ganar las elecciones. ${ }^{4} \mathrm{De}$ hecho, Carey y Siavelis muestran que las posiciones políticas dentro de la administración pública se dan a menudo como premios de consuelo a los que están dispuestos a correr bajo la bandera de la coalición en los distritos electorales de riesgo (Carey y Siavelis, 2005). En lugar de una medida para construir y mantener las coaliciones, el cuoteo se ha ganado una reputación como una forma de construcción de acuerdos corrupto. Lo que es más grave, desde el nivel del gabinete (y especialmente los últimos gabinetes de la Presidenta Bachelet) hasta el nivel de la administración pública, el cuoteo ha sido culpa de la incompetencia del gobierno, dada la falta de preparación de los funcionarios nombrados por razones partidistas. En noviembre de 2008, varios políticos de derecha pidieron la eliminación del cuoteo en el Ministerio de Salud debido a una serie de errores e irregularidades en los hospitales nacionales (Renovación Nacional, 2008).

Así, aunque el cuoteo surgió como un instrumento informal acertado para manejar la construcción y mantenimiento de la coalición durante la transición democrática, este enclave de transición se ha convertido en un impedimento para el establecimiento de patrones más representativos de reclutamiento político en Chile, con importantes consecuencias en la capacidad de los ciudadanos de hacer responsables a sus autoridades. Tal vez lo más preocupante es que existen fuertes desincentivos entre los políticos para eliminar este enclave, por la centralidad que ha tenido en el mantenimiento de la exitosa coalición a menudo difícil por la combinación de presidencialismo y multipartidismo. Sin estos tipos de acuerdos de reparto del poder será difícil continuar operando como una coalición, es decir, los partidos (ninguno de los cuales es actualmente capaz de generar apoyo mayoritario) tendrían que gobernar solos, con todas las dificultades que esto conlleva. Además, el cuoteo también 
es reforzado por la existencia de un sistema de elección parlamentario que, como será explorado a continuación, obliga a los partidos a establecer alianzas electorales para ganar. La repartición en el Poder Ejecutivo está vinculada a una dinámica más amplia de acuerdos dentro de la coalición que se encuentra relacionada con las candidaturas presidenciales y las listas legislativas conjuntas. La negociación del cuoteo y el mantenimiento continuado del mismo es uno de los factores más importantes en ayudar a mantener esta alianza electoral unida generando así fuertes desincentivos para su eliminación.

\section{Control de la elite en la selección de candidatos y la política electoral}

El proceso de selección de candidatos y la dinámica en torno a la política electoral son el segundo enclave de transición. Si bien el sistema electoral en sí fue heredado por el gobierno de Pinochet y es un enclave autoritario, la dinámica política con respecto a la negociación intrapartidaria y la selección de candidatos fue una respuesta a la elección del sistema, que fue profundamente condicionado por la transición y, por tanto, es considerado como un enclave de la transición.

El sistema de elecciones legislativas, conocido como el sistema binominal, establece dos escaños por distrito para las elecciones al Congreso, por lo que cada coalición puede presentar dos candidatos. Los detalles del sistema electoral se han analizado en profundidad en otros trabajos, por lo que no necesita ser detallado (Navia, 2005; Siavelis, 2002; Rabkin, 1996; Valenzuela y Scully, 1997). Sin embargo, en términos de la capacidad representativa del sistema electoral, la característica más importante es que la coalición con una mayor votación dentro de un distrito sólo puede ganar ambos escaños si duplica el total de la votación del segundo lugar de la lista, de lo contrario, cada una de las listas gana un escaño. Por lo tanto, en el contexto de Chile postautoritario, con un modelo de competencia de dos coaliciones, una coalición debe obtener el $66 \%$ de los votos para ganar los dos escaños, pero normalmente puede ganar un puesto con sólo el 33\%. Dado que las dos grandes alianzas casi invariablemente obtienen entre el 33\% y $66 \%$ en cada distrito, los resultados de las elecciones son una conclusión prevista: salvo en algunos casos, es probable que un miembro de la Concertación y un miembro de la Alianza ganen un escaño cada uno en cada distrito.

Si bien la democratización de los procesos de selección de candidatos tiene la posibilidad de reducir el control de la élite, la dinámica del sistema electoral también ha impedido la democratización de cualquier proceso de selección de candidatos. El control de la elite partidaria en la selección de candidatos es un proceso fundamental para el mantenimiento de la coalición. Debido a que el sistema electoral sólo proporciona dos escaños por coalición, y dado que la Concertación se encuentra integrada por importantes partidos e históricamente numerosos, el número de candidatos que cada partido recibe es objeto de arduas negociaciones previas a la elección. La complejidad de este proceso necesita de coordinación estratégica que sólo puede ser manejada, efectivamente, por las elites.

Sin embargo, la realidad de que la ubicación en la lista también es fundamental, esto lleva a que el proceso de selección esté aun más definido en las manos de las elites. Todos los actores partidarios saben que es poco probable que cualquier coalición pueda "doblar", 
o ganar dos de los dos escaños en un distrito, por lo que uno de los dos candidatos de cada coalición probablemente vaya a perder la elección. Por lo tanto, los partidos tratan de colocar a sus candidatos en la misma lista, ya sea con un candidato sumamente débil (que pueda ser derrotado fácilmente) o con un candidato extremadamente fuerte (que puede llevar la lista a una improbable victoria de dos escaños). Las negociaciones son más complicadas porque los partidos pequeños quieren ser no solo colocados en las listas sino que en las listas donde puedan ganar. Es probable que los representantes de los partidos principales derroten a candidatos de pequeños partidos, haciendo a los pequeños partidos demandar incluso compañeros débiles de lista. Esta complejidad y el manejo político implicado en la ubicación de candidaturas a listas individuales dejan la selección de candidatos completamente en las manos de elites de partido, y trabajan en contra de cualquier esfuerzo para democratizar el proceso de selección de candidatos legislativos, que ha sido dominado por las elites políticas desde el retorno a la democracia (Navia, 2008; Siavelis, 2002). La complejidad de este ambiente de negociación es un juego multinivel donde las consideraciones del mantenimiento de la coalición entran en juego y hace necesario la intervención decisiva si es que los partidos quieren alcanzar sus objetivos. Los líderes partidarios son mucho más influyentes que las bases políticas en la elección de parlamentarios. Los incentivos ofrecidos por el sistema binominal hacen que una reforma del proceso de selección de candidatos prácticamente sea imposible dado el actual patrón de competencia partidista.

Se podría argumentar que los votantes podrían recuperar su control desbancando a los incumbentes designados por las elites partidarias. Sin embargo, el sistema binominal también hace casi imposible la derrota de los incumbentes. Salvo que exista incompetencia o una indisciplina casi extrema, los partidos chilenos consideran a los titulares para tener el derecho de una nueva nominación (Siavelis, 2002). El sistema de elección, en el contexto de dos fuertes coaliciones, limita fuertemente la capacidad de derribar a un incumbente. De hecho, es poco probable que una lista contenga a dos candidatos del mismo partido, proporcionando a los incumbentes el lujo de no afrontar la competencia intrapartidaria, al menos en la arena electoral. Lo que es más importante es el hecho de que si un votante quiere derribar a un incumbente cuenta con dos estrategias potenciales. El votante puede abandonar sus convicciones ideológicas y votar a favor de una lista de oposición, o emitir un voto más ideológicamente sincero votando por el compañero de lista del incumbente. Sin embargo, dado que los votos son sumados en la determinación de la distribución de los escaños, un voto para un candidato a una lista es, en muchos sentidos, un voto para ambos candidatos. ¡Por lo tanto, votando por un compañero de lista de un incumbente, un votante en realidad puede contribuir al apoyo del incumbente a quien quiere derrocar! (Navia, 2005). Más aun, como Navia (2005) señala, debido a los umbrales del sistema, un legislador podría bajar en su nivel apoyo del 60\% al 35\% sin perder su escaño en el Congreso. El accountability es central en la democracia. La configuración coalicional combinada con las propiedades del sistema binominal no proporciona a los votantes la oportunidad de mantener a sus representantes accountables.

Una vez más, el sistema electoral entrega fuertes y positivos incentivos para la formación de la coalición, y la selección de candidatos por la vía de las negociaciones asegura la 
representación política para todos los miembros de la coalición en el Congreso. Pese a que este enclave haya sobrevivido su utilidad y se haya transformado posiblemente en contraproducente a la representación, es difícil, si no imposible de eliminar. La simple realidad de reconciliación de los objetivos políticos de varios partidos políticos, dentro del contexto de un sistema electoral con una complejidad estratégica extraoridinaria, virtualmente garantiza la continuidad de la existencia de este enclave de la transición.

\section{Dominación de los partidos en la política}

Partidos fuertes y bien institucionalizados son actores a menudo centrales en democracias de alta calidad (Mainwaring y Scully, 1995). En efecto, partidos políticos fuertes fueron centrales en el éxito de la transición chilena, porque sólo con fuertes partidos con capacidad de disciplinar a sus miembros se podían negociar, y hacer cumplir, los acuerdos que sostuvieron la transición democrática. Sin embargo, de forma cada vez mayor, la dominación del sistema político chileno por partidos con bajos niveles de adherencia popular está contribuyendo en el desarrollo de una partidocracia. Este argumento puede ser una sorpresa para quienes han seguido la cobertura de la erosión progresiva en el apoyo a los partidos entre la población chilena, tanto en la prensa y en la literatura académica (Luna, 2008; Rodríguez, 2006). Por cierto, mientras el apoyo popular hacia los partidos está siendo erosionado, ellos siguen siendo, en parte como una herencia de la transición democrática, los actores principales de organización en la política chilena. Es más, los partidos siguen funcionando dentro del mismo modelo que lo hicieron durante la transición, a pesar del hecho que la población gradualmente ha abandonado la identificación con ellos. En este sentido, y como Luna (2008) ha argumentado, existe un sistema de partido sumamente institucionalizado y estable a nivel de las elites, pero éstos tienen vínculos muy débiles con la población. Esto es un enclave problemático de la transición y una forma de actividad política que es también difícil de sacar. Partidos fuertes en el gobierno han suscrito una transición acertada y el fuerte control partidario continúa siendo necesario para lograr los acuerdos que han mantenido la Concertación unida.

Para entender este argumento es necesario poner el papel de los partidos políticos chilenos en perspectiva histórica. Antes del gobierno de Pinochet, los partidos eran reconocidos como los actores centrales en el sistema político, con altos niveles de institucionalización, identificación ciudadana y penetración social, hasta tal punto que eran referidos como "la columna vertebral" del sistema político chileno (Garretón, 1987: 64). ${ }^{5}$

Con el retorno a la democracia, y a pesar de los esfuerzos del gobierno de Pinochet para transformar el sistema de partidos, éste resurgió con la misma fisionomía general, y de hecho con los mismos líderes después de 17 años de autoritarismo (Valenzuela y Scully, 1997; Siavelis, 1997). Por todas las razones señaladas esta era una transición conducida por líderes partidarios y centrada en los partidos. A comienzos de la transición democrática, 17 partidos políticos (5 de los cuales podrían ser considerados como los principales: PS

5 Para una explicación del sistema de partidos chileno preautoritario ver Garretón (1983, 1987) y Scully (1992; especialmente Capítulo 5). 
-Partido Socialista; PPD-Partido por la Democracia; PDC-Partido Demócrata Cristiano; PR-Partido Radical, y el PSD-Partido Social Demócrata) se unieron para formar la coalición de centro izquierda con el fin de enfrentarse a la Alianza que representaba a la coalición de derecha (compuesta por dos partidos principales, la UDI-Unión Demócrata Independiente y RN -Renovación Nacional). Los partidos se dieron cuenta que la única manera de ganar las elecciones postautoritarias (especialmente a la luz del sistema electoral legislativo mayoritario legado de Pinochet) era unirse en una transición negociada caracterizada por la repartición del poder entre los principales partidos. Los partidos políticos construyeron una serie de instituciones formales e informales, negociadas a través de sus elites, que apuntaban a realizar una repartición del poder y asegurar la transición democrática. ${ }^{6}$

En el nivel de las elites, el sistema de partidos parece notablemente como el del periodo preautoritario, y numerosos estudios dan testimonio del grado de continuidad. Considerando la continuada posición central de los partidos podría caerse en la tentación de sostener que éstos todavía forman la "columna vertebral" de la política chilena. Sin embargo, si bien han sido la "columna vertebral" para la estructuración de la política de elites y en la transición democrática, la naturaleza de las relaciones entre la sociedad y los partidos es muy diferente de la que existía en el periodo preautoritario.

Cuando las encuestas de opinión comenzaron en el periodo inmediatamente después del retorno a la democracia en 1990, el 62,5\% de los chilenos señalaba que se identificaba con algún partido político. Hacia 1992, el número de chilenos que se autoidentificaban con partidos políticos aumentó a un $87 \%$. Desde allí, este porcentaje ha registrado una gradual disminución al punto que en el 2008 sólo el 43\% de los chilenos señaló que se identificaba con algún partido político en particular, y ninguno de estos registró un nivel de adhesión mayor al 10\%.7

Sin embargo, a nivel de elites, los partidos -y en particular las elites partidarias- siguen siendo los actores políticos más importantes en Chile. En primer lugar, los partidos son reconocidos como uno de los principales agentes de formulación de políticas. Las elites políticas en concertación con el presidente esquivan el trabajo legislativo del Congreso, acuerdan con los principales actores sociales y de veto antes que los proyectos sean presentados al Congreso para su aprobación. Los miembros del Parlamento también reconocen la posición central que tienen los líderes partidarios a la legislación. Una serie de preguntas extraídas en tres oleadas de encuestas a los miembros de parlamentos realizadas por la Universidad de Salamanca (por PELA - Proyecto de Elites Parlamentarias Latinoamericanas), pregunta si las estructuras de los partidos de los diputados "eran continuas" o "simplemente se movilizaban para elecciones". En las tres oleadas los diputados señalaron la permanente importancia estructural de sus partidos por amplios márgenes: 94,7\% (1994-1998), el 88,8\% (1998-2002) y 85,2\% (2002-2006). Con respecto al poder y la influencia de las elites partidarias en particular, Chile es el único país de los 15 que se incluyen en el estudio de PELA donde los líderes partidarios son rankeados

6 Para un completo análisis de las instituciones formales e informales y democratización en Chile ver Siavelis (2006).

7 Información del Centro de Estudios Públicos. Ver http://www.cepchile.cl/dms/lang_1/home.html 
como los más importantes por sobre los votantes y militantes de partidos en términos de que sus opiniones son tomadas en consideración por los diputados a la hora de tomar decisiones (Marenghi y Montero, 2008).

En segundo lugar, las elites partidarias son actores notablemente poderosos dentro de sus propios partidos. Como ya se ha señalado, éstas ejercen un control casi completo en el proceso de selección de candidatos legislativos y en los pocos casos donde se han desarrollado primarias, las elites partidistas han anulado las decisiones de competencia popular para satisfacer otros acuerdos relacionados con el mantenimiento de la coalición (Siavelis, 2002; Navia, 2008). En lo que concierne a la democracia interna de los partidos, los legisladores la perciben como bastante baja, aunque creciente cuando esta se mide en términos del poder e influencia de los militantes de los partidos. Durante los tres primeros periodos legislativos del gobierno democrático, el 16\% de los diputados calificó los niveles de democracia interna como "altos" o "muy altos" durante el periodo (19941998), el 31\% durante el segundo (1998-2002) y el 44,4\% durante el tercero (2002-2006). En general, entre los 15 países incluidos en el estudio PELA, Chile ocupó el tercer lugar inferior en términos de la percepción de la democracia interna, solo por sobre Argentina y República Dominicana (Rodríguez, 2008).

Por último, y como se ha detallado a lo largo de este trabajo, los partidos y las consideraciones de la identificación con los partidos son centrales en determinar qué puestos se reparten, dónde los candidatos parlamentarios compiten, y cómo los cargos del gobierno son distribuidos. En escritos sobre Venezuela pre Chávez, el país era visto como "un modelo" de estabilidad en América Latina. Coppedge sostuvo que "las instituciones que hacen de Venezuela una política estable también empañan la calidad de su democracia" (1994: 2). Coppedge notó que los partidos sumamente institucionalizados habían llegado a dominar completamente el sistema político en la forma de una "partiarquía" o partidocracia. De un modo muy similar, las instituciones y la dinámica política que permitieron una exitosa transición de Chile a la democracia también han empañado la calidad de su democracia, y muchas de éstas están vinculadas a una partidocracia en desarrollo.

Esto no quiere decir que la institucionalización de los partidos sea mala. Tal como Coppedge señala hay diferentes formas de institucionalización y de poder partidista, los partidos chilenos pueden desempeñar el papel fundamental en la democracia que han desempeñado en el pasado. En su estudio de Uruguay, Buquet y Chasquetti se refieren a la partidocracia de consensos, tomando nota de la extraordinaria fuerza de los partidos de Uruguay (2004). Sin embargo, si bien los partidos uruguayos demuestran muchas de las mismas prerrogativas que los partidos chilenos, la diferencia crucial es que, al contrario de Chile, cuentan con un extraordinario nivel de apoyo popular. Por lo tanto, mientras que en Chile los partidos son fuertes e influyentes a nivel de elite, cada vez más carecen de raíces profundas en la sociedad, que los caracterizó en el pasado y que ha sido reconocida como central para la efectiva representación partidaria. (Véase Rodríguez, 2006; Luna, 2008).

Evidentemente, este modelo de dominación partidaria puede ser considerado como un enclave. El sistema electoral ha obligado a los partidos a permanecer juntos si es que quieren tener una posibilidad de ganar, y la acción decidida para construir coaliciones y 
alianzas es central para continuar ganando. Sin elites partidarias fuertes que estructuren y hagan cumplir los acuerdos, es difícil forjar los tipos de esfuerzos de colaboración que han sido fundamentales para el mantenimiento de la Concertación.

Como se ha señalado, el sistema electoral es clave, porque la fuerza de los acuerdos de coalición es necesaria para seguir manteniendo el tipo de alianzas electorales necesarias para ganar bajo el sistema binominal. Quizás la demostración más clara de esta realidad fue la decisión del centro y la izquierda de presentarse en listas separadas en las elecciones municipales del 2008 -esta opción era solo posible debido a la existencia de un sistema proporcional que proveyó una constelación diferente de incentivos que el que se suscribe a este enclave.

Con un sistema electoral legislativo diferente es probable que el poder absoluto de las elites sea más moderado en diferentes maneras. La dinámica política de la dominación partidaria surgió de la correlación básica de fuerzas políticas, característica del periodo de transición, junto con la dinámica del sistema binominal. Existen limitaciones prácticas muy fuertes para reformar este enclave, no menor es el potencial desastre electoral, para los partidos y sectores ideológicos más grandes, si las negociaciones para armar listas conjuntas fallan.

\section{Formulación de políticas elitistas y extrainstitucionales}

Las elites han dominado el proceso de formulación de políticas en el periodo postautoritario, y dada la debilidad del Congreso, las negociaciones, en su mayoría, se realizan entre las elites del Ejecutivo, la oposición y actores extraparlamentarios. El modelo también incluye un acuerdo informal conocido como partido transversal, que se refiere al grupo informal integrado por políticos claves, en los primeros gobiernos democráticos, quienes se definen a sí mismos, más bien como "Lideres de la Concertación", que como líderes de sus partidos. A pesar de la falta de organización formal o encuentros formales de partidos, los propios actores saben quiénes son, y que estructuran relaciones informales entre sí, entre sus partes y la coalición, y tal como se describe más adelante, con los actores sociales cuya entrada ha sido central para el éxito legislativo de los presidentes. Ignacio Walker, quien se desempeñó en el Ministerio de la Presidencia (SEGPRES), bajo el Presidente Patricio Aylwin, señala que los miembros del partido "corresponden a una red informal que ha desarrollado una fuerte influencia bajo las tres administraciones de la Concertación, en base a un diseño estratégico y al set de políticas públicas que han llevado a cabo". (Walker, 2004: 5) Las políticas, en gran parte, han sido creadas por estas elites que trabajan en el Poder Ejecutivo.

El modelo de formulación de políticas postransición también involucra una serie de acuerdos entre el partido y las elites del Poder Ejecutivo dentro de la Concertación; y entre la Concertación y los potenciales actores de veto de la derecha, externos al Congreso. En cuanto a la relación entre la Concertación y los actores de veto de la derecha, la negociación incluye un acuerdo tácito donde el presidente puede negociar con poderosos actores económicos y líderes de la derecha para alcanzar soluciones consensuadas sobre temas de legislación más controversiales, antes que éstos sean presentados al Congreso. Este modelo, 
apodado como "democracia de los acuerdos", ha sido utilizado para realizar reformas tan importantes como el Código Tributario, la expansión del bienestar social, la legislación anticorrupción, y las extensas reformas constitucionales del año 2005. No obstante, estos grandes acuerdos de política involucraron poco y nada al Congreso y la ciudadanía (Ver Silva, 1992; Boylen, 1996).

Una vez más, este enclave de la transición fue crucial para el éxito de la transición democrática. Dado el número de partidos involucrados en la coalición el partido transversal, se facilitó el acuerdo entre los partidos. Junto con lo anterior, la fragilidad de la transición durante los primeros años de democracia, la naturaleza controversial de muchos temas políticos y la existencia de actores de veto hicieron que las negociaciones directas con estos actores haya sido una estrategia muy inteligente. Sin embargo, al pasar los años, esta política de la acomodación de elites ha creado una percepción entre la ciudadanía de que las preferencias del ciudadano importan poco, y que las políticas son un negocio más que un juego de representación. Finalmente, mediante la negociación directa con los actores sociales externos al Congreso, los presidentes han ignorado, constantemente, la principal institución de representación, lo que ha generado una percepción (incluso entre los legisladores) de que el Presidente pasa por alto el Congreso. (Siavelis, 2000). El carácter elitista y extrainstitucional de las negociaciones surgió del imperativo dual de mantener la coherencia de las políticas para una coalición presionada a competir en conjunto para ganar las elecciones, y de mantener una forma de democracia consensuada que, de lo contrario, podría producir una reacción negativa de los potenciales actores de veto. Este imperativo permanece y, una vez más, es reforzado por el sistema de elección y la dinámica de la competencia partidista, haciendo que la reforma de este enclave sea totalmente difícil de hacer.

\section{Intocabilidad del modelo económico}

Aun más que su modelo político, el modelo económico de Chile ha sido elogiado en todo el mundo por su éxito, con altas tasas de crecimiento económico e impresionantes logros en la eliminación de la pobreza. Sin embargo, el modelo económico del país sigue siendo un tema polémico. El cuestionamiento sobre las raíces del éxito económico y donde tiene lugar el crédito o la culpa, siguen siendo divisivos en Chile. Para los partidarios de Pinochet, fue su modelo económico liberal el que transformó a Chile en el libre mercado dinámico que es hoy día, y que los gobiernos de la Concertación han sabido manejar ese éxito sin alterar el modelo de Pinochet. Por otro lado, muchos críticos de Pinochet reconocen que él dirigió al país hacia el rumbo económico que tiene hoy, pero son más críticos del proceso de reforma y de sus resultados. Los críticos afirman que los gobiernos de la Concertación han mejorado el modelo imperfecto que se heredó de Pinochet, pero que los fundamentos del modelo permanecen intactos. Además, argumentan que, a pesar de los impresionantes indicadores macroeconómicos, Chile es uno de los países más desiguales en el mundo, y que la exhaustiva privatización de la salud, educación y los sistemas de seguridad social que se realizaron durante el gobierno de Pinochet han creado un eficaz sistema de dos niveles donde aquellos que tienen acceso a la privatización de bienes sociales gozan de mayores niveles de calidad y acceso. 
En esencia, los actores políticos de todo el espectro concordaban que parte del acuerdo no escrito aseguraba que en la transición el modelo económico neoliberal, heredado de Pinochet, debería permanecer sin modificaciones en sus fundamentos (Silva, 1992). Tempranamente los líderes democráticos reconocieron que la economía era el talón de Aquiles de la transición. Un cambio sustancial en la economía había ocurrido, y la integridad de la transición democrática podría haberse visto comprometida. El compromiso de los presidentes de dejar intacto el modelo económico tranquilizó a las poderosas elites económicas cuya reacción a un posible cambio en la política podría haber sido extraordinariamente desestabilizadora. Inversionistas internacionales fueron asegurados de la estabilidad económica y de un panorama confiable de inversión. Finalmente, el proceso de consulta de actores de veto sobre la economía facilitó un acuerdo posterior más amplio sobre otros asuntos con los grupos que simpatizaban con la derecha. En suma, este enclave, al igual que los anteriores, fue central en el mantenimiento de la transición democrática. Sin embargo, la falta de voluntad de los gobiernos democráticos para poner en discusión los fundamentos del modelo económico (más allá de algunas pequeñas reformas parciales, como el plan AUGE - una reforma limitada del sistema de salud emprendida por el Presidente Ricardo Lagos para hacer frente a las más graves desigualdades en el sistema de salud) ha dejado a la Concertación abierta a las críticas de que nada ha cambiado con la democracia. A pesar del éxito en la lucha contra la pobreza, los niveles de desigualdad en Chile se encuentran entre los más altos del mundo, y las debilidades en la representación ciudadana, subrayadas en este trabajo, dejan a los ciudadanos con escasas posibilidades de influir en la política económica del país. Una vez más, las negociaciones de alto nivel, en desmedro de las consultas populares o legislativas, han sido la norma en la construcción de la política económica.

El sistema político muestra signos de insatisfacción con la falta de cambios económicos. La campaña presidencial de la Presidenta Bachelet se basó en un programa de inclusión social. Sin embargo, pese a que en el discurso se indique lo contrario y de la creación de un número importante de comisiones ciudadanas extensamente convenidas para demostrar su existencia, la Presidenta continúa manteniendo los patrones elitistas de formulación de políticas que han caracterizado toda la transición. La afirmación de Bachelet de estar en contacto con las demandas reales de la gente con respecto a sus necesidades económicas y sociales fue rápidamente desmentida a principios de su administración. De hecho, la desigualdad persistente en el sistema educativo se convirtió, para la recién elegida Bachelet, en la primera gran crisis de su gobierno el que termina abruptamente el periodo de luna de miel de su administración. En mayo de 2006, y sólo con semanas de mandato, pequeñas protestas de estudiante comenzaron en respuesta a un aumento del precio en las pruebas de ingreso para la selección de estudiantes universitarios y por rumores sobre limitaciones en la obtención del pase de transporte gratuito para los estudiantes. Rápidamente las protestas aumentaron centrándose en la desigualdad y el mal estado de la educación pública en general. Se estima que medio millón de estudiantes participaron en las primeras protestas, y estas aumentaron llegando a principio de junio por sobre los 700.000 estudiantes producto de la agrupación entre estudiantes secundarios, universitarios y padres. A lo largo de 2006 y 2007 continuaron los paros y protestas estudiantiles que derivaban, a menudo, en violencia y cientos de detenciones. Bachelet y su gobierno no 
pudieron captar la naturaleza y el alcance de la insatisfacción con la economía, como de costumbre en el país.

La falta de voluntad o incapacidad de los gobiernos para hacer frente a las graves desigualdades y la demanda por generar pautas más inclusivas de políticas económicas han contribuido a la pauta general de insatisfacción mencionadas aquí. Las recientes protestas estudiantiles y disturbios labores son los primeros indicadores de descontento público con la inequidad y la falta de reformas económicas fundamentales. Políticas pragmáticas, encaminadas a abordar ahora los problemas económicos, pueden ayudar a evitar la aparición de formas más inquietantes de conflictos, que son cada vez más comunes a lo largo de América Latina.

Con esas claras señales de problemas con el modelo económico heredado, ¿por qué los gobiernos no han actuado más agresivamente para transformar sus aspectos fundamentales? Una vez más, el proceso de formulación de políticas económicas desde el retorno de la democracia se ha basado en dos tipos de acuerdos tácitos, que son enclaves de la transición, y que se fundamentan en la interacción de los partidos políticos y el contexto del sistema electoral. El primero es un acuerdo tácito entre la Concertación y la Alianza. La Concertación se ha comprometido a preservar las estructuras de seguridad económica y social creadas por la dictadura de Pinochet. Pese a que los gobiernos de la Concertación, por ejemplo, han aumentado el gasto fiscal sobre la política social en forma considerable, no han tocado, de forma alguna, ninguna de las estructuras privatizadas de asistencia sanitaria, pensiones y no han intentado ninguna forma de redistribución que pudiese nivelar la desigual estructura de distribución de ingresos o de oportunidades educacionales. Ellos han mantenido el estado de actividades económicas en la medida de lo posible, excluyendo la discusión o la puesta en práctica de cualquier clase de estrategia de desarrollo. Lo que es más, incluso en la actualidad, la Concertación debe evitar cargos de irresponsabilidades en las políticas económicas, o populismo, y de falta de voluntad en llevar a cabo transformaciones económicas fundamentales, que estan profundamente vinculadas a una habitual aversión al riesgo de parte de la elite de los grupos políticos. El segundo acuerdo se encuentra dentro de la Concertación. Como se señaló en repetidas ocasiones, el sistema electoral parlamentario obliga a la Concertación a correr como una coalición que exige, al menos, una política de consenso limitado. Participar en reformas económicas estructurales fundamentales arriesga una fractura dentro de la Concertación entre sus diversos sectores ideológicos, con resultados desastrosos, ya sea para el centro o la izquierda, dada la dinámica de un sistema electoral parlamentario que excluye, por lo menos, un sector ideológico en el contexto de tres bloques en competencia.

\section{CONCLUSIONES}

Este trabajo argumenta que un conjunto de enclaves de la transición, originados en una transición democrática, continúan dando forma a la política, incluso después del fin de la transición democrática. Estas instituciones, creencias y formas de hacer las cosas se han fusionado en enclaves identificables que son muy difíciles de desarraigar. Este análisis 
ha demostrado que, en muchas formas, todos estos enclaves han sido funcionales y han contribuido con el éxito del modelo de transición democrática de Chile, pero, en cierto sentido, ha sido una espada de doble filo. Los enclaves, si bien han sido parte central del éxito de la transición democrática, es importante destacar que todos los enclaves explorados aquí interactúan y se combinan para ofrecer fuertes limitaciones en la representación y accountability. El éxito de la Concertación (y de hecho, el éxito de la transición democrática) está basado en un complejo acuerdo de repartición de poder; pero es uno que incrementa cada vez más el poder de nominación de la elite y de políticas por medio de cuotas.

A nivel del sistema, la dinámica de interacción de coaliciones políticas y el sistema electoral proporcionan fuertes incentivos para la formación de coaliciones, pero en este proceso se ha provisto a las dos principales coaliciones de un eficaz bloqueo del poder, donde las preferencias de los ciudadanos significan poco. Cada gran coalición se asegura la efectiva garantía de uno de los dos escaños en cada circunscripción electoral. El reparto de los beneficios electorales, a través de negociaciones de asignación de candidaturas legislativas, garantizan la paz entre los partidos chilenos, pero esto sólo puede llevarse a cabo mediante la selección de los candidatos por medio de las elites, excluyendo la significativa participación por parte de los ciudadanos. Los partidos altamente institucionalizados de Chile están acreditados con la aseguración del éxito de la transición democrática y la estabilidad de la democracia chilena. No obstante, mientras la institucionalización de los partidos ha generado presidentes con mayorías legislativas, partidos fuertes y poderosos líderes partidarios, las elites de partido dominan la toma de decisiones y la selección de candidatos con poco aporte de la ciudadanía. Las elites partidarias ejercen un fuerte control sobre el comportamiento legislativo. En relación al proceso de formulación de políticas, las elites políticas, en concertación con el presidente, esquivan el trabajo legislativo, estableciendo acuerdos previos con los principales actores sociales y de veto antes de que ellos sean presentados al Congreso. Esto fue ciertamente, un fenómeno de estabilización, pero que soslaya al Congreso y la ciudadanía. Cuando se produjeron los cambios económicos, las elites, evitando los cambios desestabilizadores, han sido reacias a abordar la profunda insatisfacción pública al no comprometerse a realizar reformas sobre cualquiera de los fundamentos del sistema económico inherente a Pinochet.

Si es tan contraproducente, ¿por qué estos enclaves han permanecido? Es importante entender que la persistencia de los enclaves en Chile están basados en una lógica política concreta, sostenida por dos principales variables que se encuentran interrelacionadas: el sistema electoral binominal y la estructura de competencia partidaria postgobierno autoritario. Fundamentalmente por la competencia de múltiples partidos, que para ganar bajo el sistema binominal deben formar alianzas preelectorales. Estas alianzas preelectorales, a su vez, imposibilitan las candidaturas presidenciales de un solo partido, fuera de coalición. Desde una perspectiva política es necesaria cierta construcción de consensos y la negociación de políticas públicas. Desde una perspectiva práctica, este complejo acuerdo de reparto de poder en la elite, el gabinete y a nivel electoral solo puede ser negociado por los actores políticos poderosos. Estos actores también tienen mucho que ganar si continúan dependiendo de los enclaves de la transición para mantenerse en el poder. Mientras persistan las condiciones subyacentes que condujeron a la formación y el 
mantenimiento de los enclaves autoritarios, aquellos enclaves probablemente permanecerán con consecuencias negativas para la representación.

Existen otras variables que son menos tangibles que también contribuyen a la mantención de esos enclaves. Sin duda, el modelo de transición política fue un éxito, y la regularización de los patrones, que han sido exitosos en el pasado, tienden a ser reproducidos. Hasta que un nuevo modelo político sea concebido, las elites continuarán dependiendo de lo que ha funcionado en el pasado. La Concertación nunca ha sido derrotada en elecciones nacionales, y no ha tenido ocasión de reflexionar sobre un modelo que, hasta ahora, sea lo suficientemente eficaz para conquistar votantes. Casi la totalidad de la generación gobernante de elite política fue forjada durante el periodo preautoritario y profundamente formada por el proceso de la transición democrática, mientras que la mayoría de la masa pública nació después de que Pinochet llegara al poder. El espectacular triunfo de la oposición democrática sobre Pinochet ha proporcionado un discurso fundamental sobre la cual la Concertación ha basado sus gobiernos. El problema es que, mientras las elites chilenas miran hacia atrás, la ciudadanía chilena está a la espera de un modelo postransición de política que se despoje de estos enclaves de la transición.

¿Qué significa la persistencia de los enclaves de la transición para la democracia chilena? Este análisis ha vinculado la emergencia y perseverancia de enclaves al sistema electoral y a la relación de los patrones de competencia partidaria postransición. Por lo tanto, para derribar estos enclaves un buen punto de partida sería una reforma significativa en el sistema electoral. Aunque los cuatro presidentes del periodo postautoritarismo han presentado reformas electorales al Congreso, ninguno ha tenido éxito, tanto por los afianzados intereses de la Concertación como por la falta de voluntad de la oposición. Los incentivos políticos de corto plazo influyen en forma contraria a la reforma. Sin embargo, la introducción de alguna reforma moderada, como un sistema de representación proporcional de pequeña magnitud, podría generar mayor competencia y accountability y derribar el bloqueo que los partidos tienen sobre las nominaciones. La reforma al sistema electoral en sí no arregla todo. La capacidad representativa del sistema político se ha configurado de otras maneras por las consecuencias de estos enclaves de transición. El tradicional rol positivo de partidos efectivos y representativos en Chile ha sido, quizás, irreparablemente dañado.

Por lo tanto, es difícil imaginar una representación sustantiva de alguna otra forma que no sea a través de los partidos políticos. De hecho es irónico ver cuán efectivos fueron los partidos en el pasado, los cuales vieron disminuir su eficacia por la experiencia de gobierno autoritario, y aun más, con la adhesión a un modelo de transición político poco representativo, que ha servido para mantener la transición pero que está socavando la profundización de la democracia. Los partidos chilenos deben recuperar algo del apoyo y niveles de identificación con los que contaban históricamente intentando reconectarse con la ciudadanía. Sin embargo, mejorar la capacidad representativa de los partidos es una tarea multidimensional y, cuando la reforma electoral se realice, si es que se realiza, serán necesarios esfuerzos adicionales para aminorar el daño colateral producido por los enclaves de la transición. Nuevas formas de conexión entre los partidos y la ciudadanía, un mayor poder real para los legisladores en el proceso legislativo, mejorar los niveles 
de democracia interna de los partidos y un debate real sobre el modelo económico futuro de Chile, son todos elementos de una muy necesaria y nueva forma de políticas postransicionales que garantizan la democracia en todas sus dimensiones, incluyendo representación, legitimidad y accountability.

\section{REFERENCIAS}

Altman, David. 2006. "(Algunas) Reformas institucionales para el mejoramiento de la calidad de la democracia en Chile del siglo XXI". En Desafíos Democráticos. Editado por C. Fuentes and A. Villar. Santiago: LOM Ediciones - FLACSO, 2006, 49-85.

Boylen, Delia. 1996. "Taxation and Transition: The Politics of the 1990 Chilean Tax Reform", Latin American Research Review 31(1): 7-31.

Buquet, Daniel and Daniel Chasquetti. 2004. "La Democracia en Uruguay: Una partidocracia de consenso", Politica 42 (Fall): 221-247.

Carey, John M. and Peter M. Siavelis. 2005. "Insurance for Good Losers and the Future of the Concertación", Latin American Politics \& Society 47:2 1-22.

Coppedge, Michael. 1994. Strong Parties and Lame Ducks. Stanford, CA: Stanford University Press, 1994.

Fernández, María de los Ángeles. (n.d). "Concertación: artistas de un debate obligado", unpublished manuscript.

Garretón, Manuel Antonio. 2003. Incomplete Democracy. Chapel Hill: University of North Carolina Press, 2003.

1987. Reconstruir la política: Transición y consolidación democrática en Chile. Santiago: Editorial Andante.

1983. El proceso político chileno. Santiago: FLACSO.

Goertz, Gary. 2005. Social Science Concepts: A User's Guide. Princeton: Princeton University Press.

Helmke, Gretchen and Steven Levitsky. 2004. "Informal Institutions and Comparative Politics: A Research Agenda". Perspectives on Politics 2 (4): 725-740.

Linz, Juan. J. and Alfred Stepan, A. 1996. Problems of Democratic Transition and Consolidation: Southern Europe, South America, and Post-Communist Europe. Baltimore: The Johns Hopkins University Press.

Luna, Juan Pablo. 2008. "Partidos políticos y sociedad en Chile. Trayectoria histórica y mutaciones recientes". En Reforma de los partidos politicos en Chile. Editado por A. E. Fontaine. Santiago, Chile: CIEPLAN, 2008.

Mainwaring, Scott and Timothy Scully, eds. 1995. Building Democratic Institutions: Party Systems in Latin America. Palo Alto: Stanford University Press.

Marenghi, Patricia and Mercedes García Montero. 2008. "The Conundrum of Representation". En Politicians and Politics in Latin America. Editado por Manuel Alcántara Sáez,Boulder: Lynne Reinner, pp. 29-64.

El Mercurio. 2000. "La 'dedocracia' de Lagos", 12 March 2000, El Mercurio on line edition (www.emol.cl).

Navia, Patricio. 2008. "Legislative Candidate Selection in Chile". En Pathways to Power: Political Recruitment and Candidate Selection in Latin America. Editado por Peter M. Siavelis and Scott Morgenstern. University Park: Pennsylvania State University Press, pp. 92-118

2005. "La transformación de votos en escaños: leyes electorales en Chile, 1833-2003". Política y Gobierno 12 (2): 233-276.

Rabkin, Rhoda. 1996. "Redemocratization, Electoral Engineering, and Party Strategies in Chile, 1989-1995". Comparative Political Studies. 29(3): 335-356.

Rehren, Alfredo. 1992. "Organizing the Presidency for the Consolidation of Democracy in the Southern Cone". Ponencia presentada en la XVII International Congress of the Latin American Studies Association, Los Angeles, September 24-27, 1992, p. 7. 
Renovación Nacional. 2008. "Parlamentarios piden terminar con el cuoteo en el MINSAL", Renovación Nacional website, Noviembre 03, 2008, http://rn.cl/2008/11/03/parlamentarios-piden-terminarcon-el-cuoteo-en-el-minsal/.

Rodríguez Ruiz, Leticia M. 2006. “El sistema de partidos chileno: ¿hacia una desestructuración ideológica?" En Chile: política y modernización democrática. Editado por Manuel Alcántara and Leticia M. Ruiz Rodríguez. Barcelona: Edicions Bellaterra, pp. 73-110.

Rodríguez Ruiz, Leticia M. 2008. "The Organization of Political Parties", in ed. Manuel Alcántara Sáez and, Politicians and Politics in Latin America. Boulder: Lynne Reinner, 2008, pp. 113-138.

Scully, Timothy R. 1992. Rethinking the Center: Party Politics in Nineteenth and Twentieth Century Chile. Stanford: Stanford University Press.

Siavelis, Peter M. 2006. "Accommodating Informal Institutions and Democracy in Chile". En Informal Institutions and Democracy: Lessons from Latin America. Editado por Gretchen Helmke and Steven Levitsky. Baltimore: Johns Hopkins University Press, pp. 33-55.

2002. "The Hidden Logic of Candidate Selection for Chilean Parliamentary Elections", Comparative Politics 34 (2): 419-38.

2000. The President and Congress in Post-Authoritarian Chile. University Park, PA: Pennsylvania State University Press.

1997. "Continuity and Change in the Chilean Party System: On the Transformational Effects of Electoral Reform". Comparative Political Studies. 30(6): 651-674.

2008. "Elite-Mass Congruence and the Quality of Democracy in Chile" ponencia presentada en la 2008 Annual Meeting of the American Political Science Association, Boston, Massachusetts, August 28-31, 2008.

Silva, Eduardo. 1992. "Capitalist Regime Loyalties and Redemocratization in Chile". Journal of Interamerican Studies and World Affairs 34(4): 39-76.

La Tercera. 2001. "Presiones por los cupos de subsecretarios", 1 febrero 2000, La Tercera_on line edition (www.latercera.cl).

Valenzuela, Samuel and Timothy R. Scully. 1997. "Electoral Choices and the Party System in Chile: Continuities and Changes at the Recovery of Democracy", Comparative Politics 29 (4): 511-27.

Walker, Ignacio. 2004. "Chile: Three Stories of Informal Institutions in a Limited Democracy", ponencia presentada en la conferencia, Informal Institutions and Politics in Latin America, University of Notre Dame, South Bend, ID, April 23-24, 2003.

Peter M. Siavelis es Profesor Asociado de Ciencia Política en Wake Forest University, EE.UU. Es autor de The President and Congress in Post-Authoritarian Chile: Institutional Constraints to Democratic Consolidation (Penn State, 2000) y codirector (con Scott Morgenstern) de Pathways to Power: Political Recruitment and Candidate Selection in Latin America (Penn State, 2008). Su trabajo ha aparecido en Comparative Politics, Comparative Political Studies, Estudios Públicos, Latin American Politics and Society, Latin American Research Review, Party Politics, Política y Revista de Ciencia Política, entre otras.

[E-mail: siavelpm@wfu.edu] 
